\title{
On Extending Symmetry Sets for 2D Shapes
}

\author{
Arjan Kuijper and Ole Fogh Olsen \\ Image group, IT-University of Copenhagen \\ Glentevej 67, DK-2400 Copenhagen, Denmark
}

\begin{abstract}
Many attempts have been made to represent families of 2D shapes in a simpler way. These approaches lead to so-called structures as the Symmetry Set $(\mathcal{S S})$ and a subset of it, the Medial Axes $(\mathcal{M A})$. While the latter is commonly used, the former is still in the mathematical research stage. One reason for this is that in contrast to the $\mathcal{S S}$, the $\mathcal{M A}$ can be computed efficiently and fast, and yields one connected component for a closed shape.

In this paper a natural complement of the symmetry set, called the AntiSymmetry Set $(\mathcal{A S S})$, is used to connect components bearing the full richness of the symmetry set. Secondly, new ways are presented to visualize these sets. One uses the radius of the describing circle as extra dimension, the other, the so-called pre-Symmetry Set (pre-SS $)$, uses the parameter space. Example shapes show the extra information carried in the $\mathcal{A S S}$ and the pre- $\mathcal{S}$ in determining the special points on the $\mathcal{S}$ as well as revealing the structure of the $\mathcal{S} \mathcal{S}$ in more detail. They are also capable of distinguishing between different shapes where the $\mathcal{S} \mathcal{S}$ and the $\mathcal{M A}$ in some cases fail.
\end{abstract}

\section{Introduction}

In 2D shape analysis the simplification of shapes into a skeleton-like structure is widely investigated. The Medial Axis $(\mathcal{M A})$ skeleton [4] is commonly used, since it can be calculated in a fast and robust way. Many resuls on simplification, reconstruction and database search are reported. The $\mathcal{M A}$ is a member of a larger family, the Symmetry Set $(\mathcal{S S})$, exhibiting nice mathematical properties, but difficult to compute and yielding distinct branches $[6,7]$. To overcome the latter we introduce the anti-symmetry set $(\mathcal{A S S})$, resulting in a connected set.

Secondly, while the $2 \mathrm{D}$ visualization of the $\mathcal{M} \mathcal{A}$ skeleton is unambiguous due to its limiting nature, the $\mathcal{S} \mathcal{S}$ may give rise to intersecting curves that sometimes occur due to projections. Together with the need for augmenting the skeleton with information of the radius / scale / distance from the boundary at which it occurs, the need for a representation in a space with an extra dimension is evident - something that was already known for the skeleton [4] and used in the so-called Shock Graph method [15]. It has been mentioned by Wright et al. [17], but not been used afterwards. Another way of representation is obtained

* This work is part of the DSSCV project supported by the IST Programme of the European Union (IST-2001-35443). 
when the $\mathcal{S S}$ is considered in the parameter space, yielding the so-called PreSymmetry Set. A reason for using the $\mathcal{S S}$ instead of the $\mathcal{M A}$ is that the $\mathcal{S} \mathcal{S}$ can be caught in a linear data structure, as presented elsewhere[12], in contrast to the graphs needed for representation of the $\mathcal{M A}$.

We will first give a short overview on the $\mathcal{M A}$ and the $\mathcal{S} \mathcal{S}$ and then introduce the $\mathcal{A S S}$ and new ways for visualization.

\section{Some Background Theory on Shapes}

In this section we give the necessary background regarding properties of shapes, the Medial Axis, the Symmetry Set, labelling points on these sets and give an example to clarify the definitions.

Let $\mathcal{S}(x, y)=\{(x, y) \mid L(x, y)=0\}$ denote a closed 2D shape. Then $\mathcal{N}(x, y)=$ $\left(L_{x}, L_{y}\right)\left(L_{x}^{2}+L_{y}^{2}\right)^{-1 / 2}$ denotes its unit normal vector, and $\kappa(x, y)=-\left(L_{x}^{2} L_{y y}-\right.$ $\left.2 L_{x} L_{y} L_{x y}+L_{y}^{2} L_{x x}\right)\left(L_{x}^{2}+L_{y}^{2}\right)^{-3 / 2}$ its curvature. The evolute $\mathcal{E}(t)$ is given by the set $\mathcal{S}+\mathcal{N} / \kappa$. Even if the curve is smooth and differentiable, the evolute contains non-smooth and non-differentiable points, viz. those where the curvature is zero or takes a local extremum, respectively.

\subsection{The Medial Axis and Symmetry Set}

The Medial Axis $(\mathcal{M A})$ is defined as the closure of the set of centers of circles that are tangent to the shape at least two points and that contain no other tangent circles: the are so-called maximal circles. The Symmetry Set $\mathcal{S} \mathcal{S}$ is defined as the closure of the set of centers of circles that are tangent to the shape at least two points $[6,5,8,7]$. Obviously, the $\mathcal{M A}$ is a subset of the $\mathcal{S} \mathcal{S}[7]$.

To calculate these sets from above definition, the following procedure can be used: Let a circle with unknown location be tangent to the shape at two points. Then its center can be found by using the normal vectors at these points: it is located at the position of each point minus the radius of the circle times the normal vector at each point. To find these two points, the location of the center and the radius, do the following: Given two vectors $p_{i}$ and $p_{j}$ (right, with $i=1$ and $j=2$ ) pointing at two locations at the shape, construct the difference vector $p_{i}-p_{j}$. Given the two unit normal vectors $N_{i}$ and $N_{j}$ at these locations, construct the vector $N_{i}+N_{j}$. If the two constructed vectors are non-zero and perpendicular,

$$
\left(p_{i}-p_{j}\right) \cdot\left(N_{i}+N_{j}\right)=0,
$$

the two locations give rise to a tangent circle. The radius $r$ and the center of the circle are given by

$$
p_{i}-r N_{i}=p_{j}-r N_{j}
$$

\subsection{Labelling Points}

It is known that the $\mathcal{M A}$ in itself carries insufficient information for representing and reconstruction a shape, since different shapes can yield the same $\mathcal{M} \mathcal{A}$. 
Therefore additional information can be added to the $\mathcal{M} \mathcal{A}$ and used, as proposed by various authors [13-16]. It has been shown by Bruce et al. [6] that only five distinct types of points can occur for the $\mathcal{S S}$, and by Giblin et al. [8,7] that they are inherited by the $\mathcal{M A}$. An $A_{1}^{2}$ point is the "common" midpoint of a circle tangent at two distinct points of the shape. An $A_{1} A_{2}$ point is the midpoint of a circle tangent at two distinct points of the shape but located at the evolute. An $A_{1}^{2} A_{1}^{2}$ point is the midpoint of two circles tangent at two pairs of distinct points of the shape with different radii. An $A_{1}^{3}$ point is the midpoint of one circle tangent at three distinct points of the shape. An $A_{3}$ point is the midpoint of a circle located at the evolute and tangent at the point of the shape with the local extremal curvature.

Based on the behaviour of the radii one can impose vectors on the $\mathcal{M A}$ denoting increasing radius, for example. This has been done in the so-called Shock Graph approach by Siddiqi et al. [13-16]. The $\mathcal{M A}$ or the $\mathcal{S} \mathcal{S}$ together with the radius function is sufficient to reconstruct the shape [7].

\section{Beyond the Symmetry Set}

In this section we present three ways to add extra information to the $\mathcal{S} \mathcal{S}$ based on Eqs. (1-2). The first is straightforward: use the radius of tangent circle to each set, as described in the previous section as an extra dimension. The second method is an extension of the $\mathcal{S S}$, called the anti-symmetry set $(\mathcal{A S S})$. Thirdly, the pre-symmetry set (pre-SS $)$ will be introduced as analysis and visualization tool. We focus on the $\mathcal{S S}$, since the $\mathcal{M A}$ is only a subset of it.

\subsection{The $\mathcal{S} \mathcal{S}$ Radius Space}

Having incorporated the radius function upon the $\mathcal{S} \mathcal{S}$ one has extra information. This information can be exploited in much more detail when the radius is considered as an extra dimension. First attempts were reported in [17], albeit only on a spline approximation of the shape. Using this dimension, the 1D curves in the $2 \mathrm{D}$ plane become $1 \mathrm{D}$ curves in $3 \mathrm{D}$ space. In this $3 \mathrm{D}$ space the $\mathcal{S} \mathcal{S}$ curves reveal information that does not appear in a trivial manner in the $2 \mathrm{D}$ plane. For example, at an $A_{1}^{2} A_{1}^{2}$ point of the $\mathcal{S S}$, two curves are intersecting in $2 \mathrm{D}$, but obviously not in $3 \mathrm{D}$, since two different radii were involved. At an $A_{1}^{3}$ point, in contrast, three curves still intersect in the 3D space.

On the other hand, points at the $\mathcal{S} \mathcal{S}$ that arise from locally minimal or maximal circles are clearly visible as local extrema of the $3 \mathrm{D}$ curve with respect to the radius.

\subsection{The Anti-symmetry Set}

Another extension is the Anti-Symmetry Set $(\mathcal{A S S})$. It is defined as the set of points satisfying Eq. 1, but not being part of the symmetry set. Figure 1b clarifies this. 
The points $p_{1}$ to $p_{4}$ have in common that they give rise to points on the $\mathcal{M A}$ or the $\mathcal{S S}$ in specific pairs. For example, $p_{1}$ and $p_{2}$ define a $\mathcal{M A}$ (and $\mathcal{S S}$ ) point and $p_{1}$ and $p_{4}$ a $\mathcal{S S}$ point. The other combination $p_{1}$ and $p_{3}$ satisfies Eq. 1, but is not part of the $\mathcal{S} \mathcal{S}$ : they are part of the $\mathcal{A S S}$.

The anti-symmetry set appeared in the early 1990's due to Blake et al. [3,2] in the field of robotics. There they considered this set in order to find an optimal finger position for a two finger grasp. Another - perhaps less striking - name is due to Giblin [11]: the Mid Parallel Tangent Locus, describing exactly what it is.

\subsection{The Pre-symmetry Set}

One way to visualize the locations the sign changes of Eq. 1 is by taking all points on the shape pair wise and plot these sign changes in a diagram. This was used by Holtom [11] and Giblin and Sapiro [9, 10,1] in a different context (affine symmetry sets). Following their line of reasoning this diagram should be called the pre- $\mathcal{S S}$. Since it consists of non-intersecting lines for the $\mathcal{S S}$ it can act as a linear data structure describing the shape. In combination with the $\mathcal{A S S}$, same intersections occur as with the $\mathcal{S S}$ [12]. In the next section we will discuss the pre- $\mathcal{S S}$ and its properties in more detail.

\section{Example Shapes}

In this section we deal with some example shapes, staring with the simplest one: an ellipse. Since the ellipse is highly symmetric, some results may seem trivial, while others give a false intuition. We therefore continue with two other examples: a "cubic oval" [5] and a concave shape.

\subsection{Ellipse}

The ellipse $L(x, y)=x^{2}+4 y^{2}-4=0$ has $\mathcal{N}(x, y)=(x, 4 y)\left(x^{2}+16 y^{2}\right)^{-1 / 2}$, and $\kappa(x, y)=-32\left(x^{2}+4 y^{2}\right)\left(4 x^{2}+64 y^{2}\right)^{-3 / 2}$, with extremal values $1 / 4$ and 2 at the four locations $(0, \pm 1)$ and $( \pm 2,0)$, respectively. The evolute consists of four parts, joined at cusps at the locations $(0, \pm 3)$ and $( \pm 3 / 2,0)$.

To find the $\mathcal{M A}$ with Eq. 2 we have two points on the shape with $y_{1}=-y_{2}$ and $x_{1}=x_{2}$ and $\tilde{y_{1}}=\tilde{y_{2}}=0$ and $\tilde{x_{1}}=\tilde{x_{2}}$ for $(\tilde{x}, \tilde{y})$ a point on the $\mathcal{M A}$. Then $y_{1}-4 r y_{1} / \sqrt{x^{2}+16 y_{1}^{2}}=y_{2}-4 r y_{2} / \sqrt{x^{2}+16 y_{2}^{2}}$ yields $r=-\sqrt{x^{2}+16 y_{2}^{2}} / 4$. Together with $y_{1,2}= \pm \sqrt{4-x^{2}}$ this gives $r=-\sqrt{16-3 x^{2}} / 4$. Then the points on the $\mathcal{M A}$ are given by $(\tilde{x}, \tilde{y}, r)=(x+r N, y+r N, r)=(x-x / 4, y-$ $\left.4 y / 4,-\sqrt{16-3 x^{2}} / 4\right)=\left(3 x / 4,0,-\sqrt{16-3 x^{2}} / 4\right)$. Note that $x \in[-2,2]$, so $\tilde{x} \in$ $[-3 / 2,3 / 2]$. The radius varies from $-1 / 2$ at the endpoints to -1 at the origin. So it has a $\mathcal{M A}$ formed by a straight line along the $x$-axis with its endpoints at the cusps of the evolute within the shape: $( \pm 3 / 2,0)$.

Similarly, one can find the expression for the $\mathcal{S S}$ to be the curve above combined with the curve $(\tilde{x}, \tilde{y}, r)=\left(0,3 y,-\sqrt{4+12 y_{2}^{2}}\right)$, which varies from $(0,-3,-4)$ via $(0,0,-2)$ to $(0,3,-4)$, since $y \in[-1,1]$, as shown in Figure $1 \mathrm{c}$. 

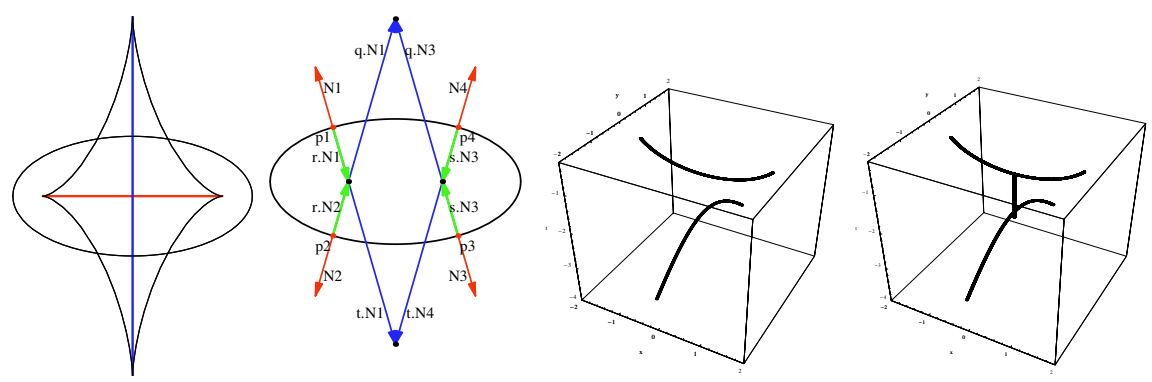

Fig. 1. a) The ellipse, its evolute and its $\mathcal{M A}$ (horizontal line) and $\mathcal{S} \mathcal{S}$ (both lines) in 2D. b) Combinations of points contribute to the $\mathcal{M A}, \mathcal{S S}$, or $\mathcal{A S S}$. c) 3D representation of the $\mathcal{S S}$. d) The $\mathcal{S S}$ and $\mathcal{A S S}$ in $3 \mathrm{D}$.
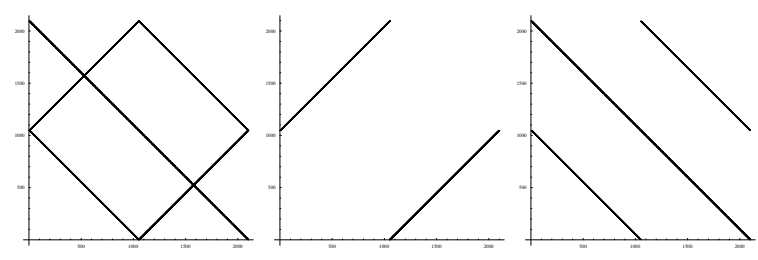

Fig. 2. The pre- $\mathcal{S}$. Left to right: all zerocrossings, the $\mathcal{A S S}$ part and the $\mathcal{S} \mathcal{S}$ part.

It therefore has a $\mathcal{S S}$ formed by the $\mathcal{M A}$, and a straight line along the $y$-axis with its endpoints at the cusps of the evolute outside the shape: $(0, \pm 3)$. So at the origin in $2 \mathrm{D}$ we have an $A_{1}^{2} A_{1}^{2}$ point, with radii 2 and 1 , see Figure 1a.

The $\mathcal{A S S}$ points are found as those points with $\left(x_{1}, y_{1}\right)=\left(-x_{2},-y_{2}\right)$ and with radii $\left.r=\sqrt{x_{1}^{2}+y_{1}^{2}}\right)$, varying between 1 and 2 . This is identical to the two radii of the $\mathcal{S S}$ at the planar origin. The pre- $\mathcal{S S}$ and the parts of it determining the $\mathcal{S S}$ and the $\mathcal{A S S}$ are shown in Figure 2 from left to right. The diagrams are symmetric in the diagonal $p_{i}, p_{i}$. The dark lines represent the zero crossings of Eq. 1. At intersections of the diagonal and a zero crossing, a branch of the $\mathcal{S} \mathcal{S}$ starts in an $A_{3}$ point. Since the shape is closed, the lines continue through the boundaries: the square represents a torus, since the starting point is arbitrary. Therefore the $\mathcal{S S}$ part contains four intersections with the diagonal, corresponding to the four cusps of the evolute. The two lines forming the $\mathcal{S S}$ of the square in the left image constitute one branch of the $\mathcal{S S}$. The long line together with the point at the origin (being a $A_{3}$ point) forms the second part of the $\mathcal{S} \mathcal{S}$. The $\mathcal{A S S}$ image connects the curves. The intersections of the $\mathcal{S S}$ and the $\mathcal{A S S}$ are local extrema w.r.t. radius of the $\mathcal{S} \mathcal{S}$, see Figure $1 \mathrm{~d}$.

\subsection{The Cubic Oval}

Firstly the closed part of a cubic oval given by $y^{2}=2 b x y+a^{2}\left(x-x^{3}\right)$, with $a=1.025$ and $b=0.09$ is taken. Then six extrema of the curvature occur, while the curvature doesn't change sign and the shape is thus convex [6]. The 

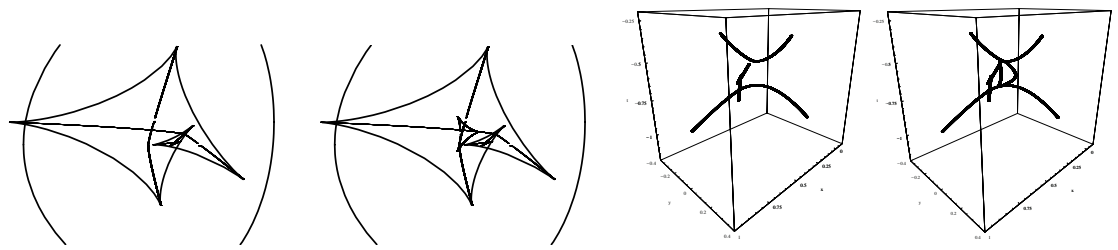

Fig. 3. Evolute of the cubic oval with the $\mathcal{S S}$ (a )and the $\mathcal{A S S}$ and the $\mathcal{S} \mathcal{S}$ (b) in 2D. Radius space with the $\mathcal{S S}(\mathrm{c})$ and the $\mathcal{A S S}$ and the $\mathcal{S S}(\mathrm{d})$.
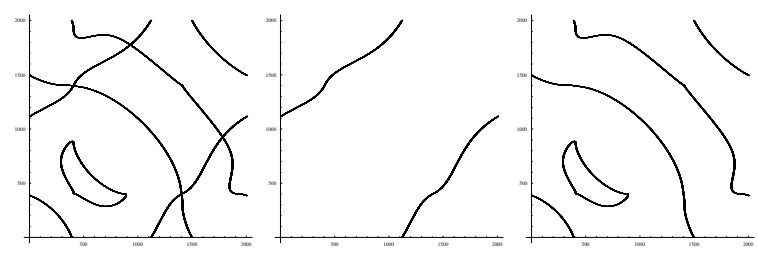

Fig. 4. The pre- $\mathcal{S S}$ of the cubic oval. Left to right: all zerocrossings, the $\mathcal{A S S}$ part and the $\mathcal{S}$ part.

extra extrema of the curvature arise from a perturbation of the shape [5]. Since they alternate along the shape, a maximum and a minimum are created. As a consequence, the evolute is self-intersecting. Furthermore, the evolute consists six cusps. A direct consequence of this in its turn is that a new branch of the $\mathcal{S S}$ is created, since these branches always start in the cusps. This branch must be essentially different from the two other branches, since the original branches start in cusps that both arise from either local maxima of the curvature, or minima. These $\mathcal{S} \mathcal{S}$ curves essentially need to have a local extremum with respect to the radius in 3D, as in Figure 1c. The newly created branch, however, has a minimum and a maximum as endpoints, so its behaviour in 3D must be different. The behaviour of the pre- $\mathcal{S} \mathcal{S}$ is also different: a new branch implies a new "line" in the pre- $\mathcal{S S}$. Since the perturbation is a local effect, not all points on the shape are involved in creation the new branch. The "line" in the pre- $\mathcal{S} \mathcal{S}$ thus must be a closed loop. This is indeed what occurs in Figure 4.

The projection of the $\mathcal{S S}$ and the $\mathcal{A S S}$ with the $\mathcal{S S}$, Figure 3, shows that again three curves joining pair wise in cusps for the ASS. The newly created branch shows some extra behaviour, as shown in Figure 5a. As clearly visible, the new branch bounces twice to the evolute, thus containing two $A_{1} A_{2}$ points. In the pre- $\mathcal{S} \mathcal{S}$ (Figure $5 \mathrm{~b}$ ) these points are visible as the local extrema in vertical or horizontal direction of the closed loop (which has four, but two are due to the symmetry along the diagonal) due to the same bouncing.

Taking a closer look at the pre- $\mathcal{S} \mathcal{S}$, one can see that the curve starting top-left also contains two pairs of local extrema. Consequently, one of the two original curves also contains two $A_{1} A_{2}$ points. This could also have been seen from the $\mathcal{S S}$ shown in Figure 5a: one curve traverses the evolute, which can only occur 

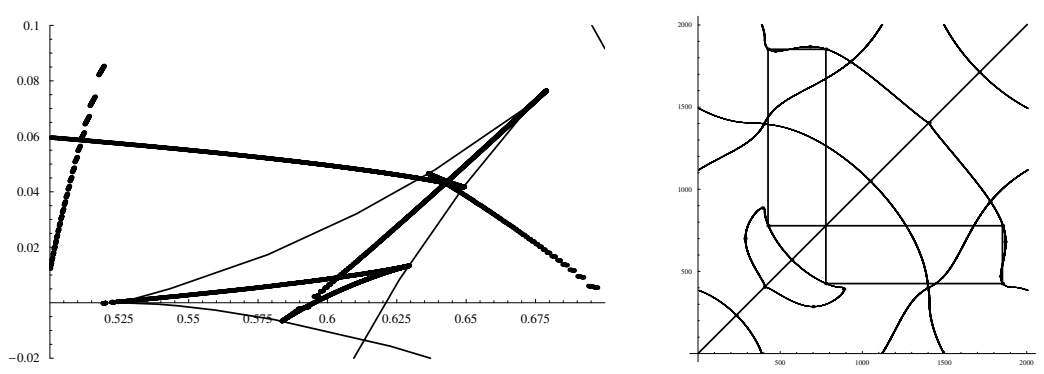

Fig. 5. Close up of the cubic oval with its evolute and symmetry set. Pre-SS $\mathcal{S}$ of the cubic oval, with special points (see text).

by a double $A_{1} A_{2}$ combination. A way to get a grip on this is by the following: Consider the manifold making the swallowtail, with the $A_{3}$ 's on the corners. The symmetry sets walks along the manifold, starting from one $A_{3}$. Then it comes to the "end" of the manifold and continues its walk along the down-under part until it comes again to an end, where it goes on along the upper part until it reaches the second $A_{3}$ corner point. The same holds for the part of the symmetry set from which this new part originated ${ }^{1}$.

As a result of the creation the new and old curve intersect in a $A_{1}^{3}$ point, where a circle is located tangent to three different points of the shape. Here three branches of the $\mathcal{S S}$ intersect. This $A_{1}^{3}$ point is also visible, albeit a bit hidden, in the pre- $\mathcal{S S}$. Figure $5 \mathrm{~b}$ shows the pre- $\mathcal{S} \mathcal{S}$ with all the special points: the $A_{1} A_{2}$ points as the local extrema in horizontal or vertical direction, the $A_{3}$ points at the intersection of a curve with the diagonal, the local extrema on the 3D $\mathcal{S S}$ curves as the intersections of the $\mathcal{S S}$ and $\mathcal{A S S}$ zero crossing curves, and the $A_{1}^{3}$ as the set of points linked by the lines. Only at a $A_{1}^{3}$ point there are three points in the pre- $\mathcal{S} \mathcal{S}$ with the combination $\left(p_{1}, p_{2}\right),\left(p_{1}, p_{3}\right)$, and $\left(p_{2}, p_{3}\right)$ (and, of course, its diagonal symmetric counterpart). The $3 \mathrm{D}$ visualization of the $\mathcal{A S S}$ and the $\mathcal{S S}$, Figure 3d, shows again this all in one plot.

\subsection{The Concave Case}

As a more complicated concave shapes the one given by the equation $\left(x^{2}+y^{2}+\right.$ $\left.a^{2}\right)^{2}=b^{2}+4 a^{2} x^{2}$, with $a=1.99$ and $b=4$ is taken. However, this example shows that the pre- $\mathcal{S S}$ for solely the $\mathcal{S S}$ part fails in determining the shape, see Figure 7: it has the same structure as the ellipse. This difference becomes clearer in Figure 6: The $\mathcal{S S}$ contains two straight lines, just as for the ellipse, albeit in this case one line "goes via infinity", since $\kappa=0$, causing the two crosses. In $3 \mathrm{D}$ the distinction between the two branches on each side going to infinity is clearly visible (Figure 6c). The upper one corresponds to a 'negative' radius, i.e.

\footnotetext{
${ }^{1}$ This is the result of unfolding [5] the $A_{4}$ that appeared when the perturbation held one double (non-generic) extremum, i.e. the transition from 0 to 2 new extremal values of the curvature of the shape.
} 

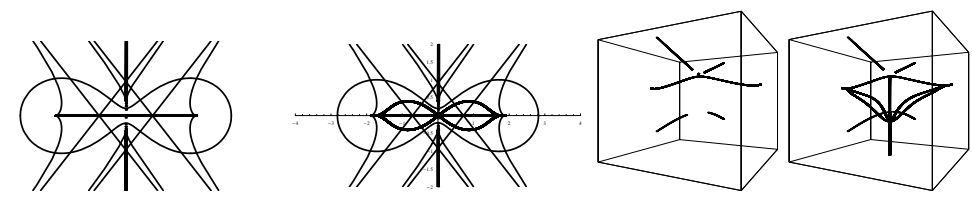

Fig. 6. Evolute of the concave shape with the $\mathcal{S S}$ (a) and the $\mathcal{A S S}$ and the $\mathcal{S} \mathcal{S}$ (b). Radius space with the $\mathcal{S S}(\mathrm{c})$ and the $\mathcal{A S S}$ and the $\mathcal{S S}(\mathrm{d})$.
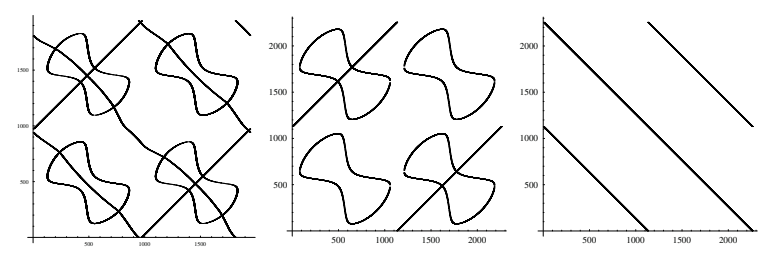

Fig. 7. Left to right: The pre-SS, the $\mathcal{A S S}$ part and the $\mathcal{S S}$ part.

the radius related to a normal pointing outwardly. So again in the $\mathcal{S} \mathcal{S}$ and $\mathcal{A S S}$ combination, the local extrema of the positive radii branches are connected. Note that the part along the $x$-axis contains three extrema.

\section{Summary}

In order to gain sufficient information from the $\mathcal{M A}$ or the $\mathcal{S S}$ additional information is needed. In this paper we extended the $\mathcal{S S}$ with information of the $\mathcal{A S S}$, the pre-SS and the visualization using the describing circle as an extra dimension. The representations in parameter space and in 3D space carry more information than the commonly used $2 \mathrm{D}$ visualization. In $3 \mathrm{D}$ possible ambiguities are avoided, while the use of the $\mathcal{A S S}$ guarantees a connection between the local extrema of the main positive radii branches, which are the main curves of symmetry. Next, we showed that special points along the $\mathcal{S} \mathcal{S}$ can also be found in the pre- $\mathcal{S S}$, that can be used as a good indicator of the complexity of the $\mathcal{S S}$ and as localization tool for determining special points on it. Using these extensions of the $\mathcal{S S}$, one is able to catch the $\mathcal{S S}$ in a linear data structure [12], dependent on the $1 \mathrm{D}$ curves in the pre- $\mathcal{S} \mathcal{S}$, in contrast to the graphs needed for representation of the $\mathcal{M} \mathcal{A}$.

\section{References}

1. S. Betelu, G. Sapiro, A. Tannenbaum, and P. Giblin. Noise-resistant affine skeletons of planar curves. In Proceedings of the 6th European Conference on Computer Vision (2000), volume 1842, pages 742-754, 2000. LNCS 1842.

2. A. Blake and M. Taylor. Planning planar grasps of smooth contours. Robotics and Automation, 1993. Proceedings., 1993 IEEE International Conference on, pages 834-839 vol.2, 1993. 
3. A. Blake, M. Taylor, and A. Cox. Grasping visual symmetry. Computer Vision, 1993. Proceedings., Fourth International Conference on, pages 724-733, 1993.

4. H. Blum. Biological shape and visual science (part i). Journal of Theoretical Biology, 38:205-287, 1973.

5. J. W. Bruce and P. J. Giblin. Growth, motion and 1-parameter families of symmetry sets. Proceedings of the Royal Society of Edinburgh, 104(A):179-204, 1986.

6. J. W. Bruce, P. J. Giblin, and C. Gibson. Symmetry sets. Proceedings of the Royal Society of Edinburgh, 101(A):163-186, 1985.

7. P. J. Giblin and B. B. Kimia. On the intrinsic reconstruction of shape from its symmetries. In IEEE Conference on Computer Vision and Pattern Recognition, 1999, pages 79-84, 1999.

8. P. J. Giblin and B. B. Kimia. On the local form and transitions of symmetry sets, medial axes, and shocks. In Proceedings of the 7th International Conference on Computer Vision (1999), pages 385-391, 1999.

9. P.J. Giblin and G. Sapiro. Affine-invariant distances, envelopes and symmetry sets. Geometriae Dedicata, 71(3):237-262, 1998.

10. P.J. Giblin and G. Sapiro. Affine invariant medial axis and skew symmetry. Computer Vision, 1998. Sixth International Conference on, pages 833-838, 1998.

11. P. A. Holtom. Affine-Invariant Symmetry Sets. PhD thesis, University of Liverpool, 2000 .

12. A. Kuijper. Computing symmetry sets from 2 d shapes, 2003. Technical report, IT University of Copenhagen, Accepted ECCV 2004.

13. M. Pelillo, K. Siddiqi, and S. Zucker. Matching hierarchical structures using association graphs. IEEE Transactions on Pattern Analysis and Machine Intelligence, 21(11):1105-1120, 1999.

14. T.B. Sebastian, P.N. Klein, and B. B. Kimia. Recognition of shapes by editing shock graphs. In Proceedings of the 8th International Conference on Computer Vision (2001), pages 755-762, 2001.

15. K. Siddiqi and B.B. Kimia. A shock grammar for recognition. Computer Vision and Pattern Recognition, 1996. Proceedings CVPR '96, 1996 IEEE Computer Society Conference on, pages 507-513, 1996.

16. K. Siddiqi, A. Shokoufandeh, S. Dickinson, and S. Zucker. Shock graphs and shape matching. International Journal of Computer Vision, 30:1-22, 1999.

17. M.W. Wright, R. Cipolla, and P.J. Giblin. Skeletonization using an extended Euclidean distance transform. Image and Vision Computing, 13(5):367-375, 1995. 